\title{
Embedded Fault Diagnosis Expert System on Weapon Equipment
}

\author{
Chaoyang Geng , Fenli Gao \\ School of Computer Science and Engineering, Xi'an Technological University, \\ Xi'an China, 710021, \\ email:541211200@qq.com
}

\begin{abstract}
Keywords: Embedded Linux; Fault diagnosis; Expert system; Agent.
\end{abstract}
\begin{abstract}
In order to improve the work performance and mobility of the fault diagnosis expert system, a portable diagnostic client for embedded Linux operating system is developed. The data acquisition module is embedded in the device by analyzing fault-tree based troubleshooting process, using the fault symptom table for fault diagnosis. Maintenance staff determines whether the equipment is working properly in accordance with the output signal of the diagnostic Agent which is embedded in weapons equipment, and infers equipment fault information by diagnosis clients, then locate the fault source quickly. Embedded fault diagnosis expert system integrates the data acquisition functions and reasoning capabilities of the knowledge base effectively, thus allowing the maintenance staff to diagnose and repair the equipment quickly and efficiently.
\end{abstract}

\section{Introduction}

The modern weapon equipment is a high technology product integrates light, machine and electricity with complex structure, high degree of information and powerful functions, it can make a correctly judgments and eliminate timely the fault of weapons and equipment in actual training and actual combat, this ensure that the weapon system survivability and reliability, which is the premise and guarantee to improve the combat effectiveness of the army [1]. But the army maintenance personnel change frequently, facing a variety of technical equipment repair contradiction notable, wherein the fault diagnosis is the bottleneck of equipment repair.

Fault diagnosis technology as a discipline originated in the 60's of the twentieth century, the related test equipment also experienced manual, semi-automatic, and then realize the process to the automation development, intelligent level of testing technology continuously improve. At present, the main testing equipment of weapons is Modular Instrument based on VXI bus, which is a virtual instrument system taking computer as the core. It can realize fast automatic detection; however, the fault location and diagnosis often need experts and technicians to participate in. The human factor plays a key role in the whole process of fault diagnosis, equipment test still need a lot of experts and technical personnel to participate in. In this way, on the one hand it would have been a very prominent issue about technical personnel more outstanding, on the other hand also influence the ability of quick response of army [2]. Fault diagnosis expert system by collecting expert knowledge, using intelligent reasoning method to accomplish the fault diagnosis process, in order to making the computer plays the role of human experts in this field, by using the expert system, common repair personnel can also imitate the expert to solve practical problems of work process, method, experience and so on others practical problems. The development trend of the modern weapon equipment fault diagnosis technology is fast, intelligent, miniaturization, self-diagnosis and integrated, in the new development of weapon equipment use artificial intelligence method, implement comprehensive diagnosis support technology, pay attention to the open architecture design of monitoring and diagnostic system as well as equipment, strict formulate new technical standards and testing standards to improve the comprehensive performance of Diagnostic equipment [3].

Embedded fault diagnosis expert system is introduced in this paper based on the platform of the ARM920T microprocessor, making the system miniaturization and the mobility is greatly improved, besides the fault diagnosis Agent will be embedded in weapon equipment is diagnosed, 
and real-time acquisition working condition of equipment, then by the connection of a client and diagnosis of Agent, it can fast implementation the reasoning and judgments, as the same time send the diagnosis results to the repair personnel, for help them quickly, accurately find fault location, in order to improve the efficiency of weapon equipment fault diagnosis.

\section{The Study of Diagnostic Methods}

The organization form of traditional expert system usually uses fault tree, in the diagnostic process requires artificial participation, the diagnostic steps are Cumbersome. As shown in fig. 1 is a separation fault tree of a equipment, when the equipment failure, first observe the power indicator light, if the power light is abnormal, suspect that power failure, check the power supply; If the power light is normal, suspect sensor cable fault, check the sensor cable; If the inspection found power failure, then repair of power; If the inspection found no fault in the power supply, power cable fault is suspect, check the power cable, and so on, fault check according to the order of this, gradually reduce the fault coverage, and ultimately to determine the source of failure. This is the equipment with relatively simple structure, for complex equipment, fault tree depth is often achieved several layer even deeper, through the fault tree to find the fault step is very complicated, so that the diagnosis inefficiency.

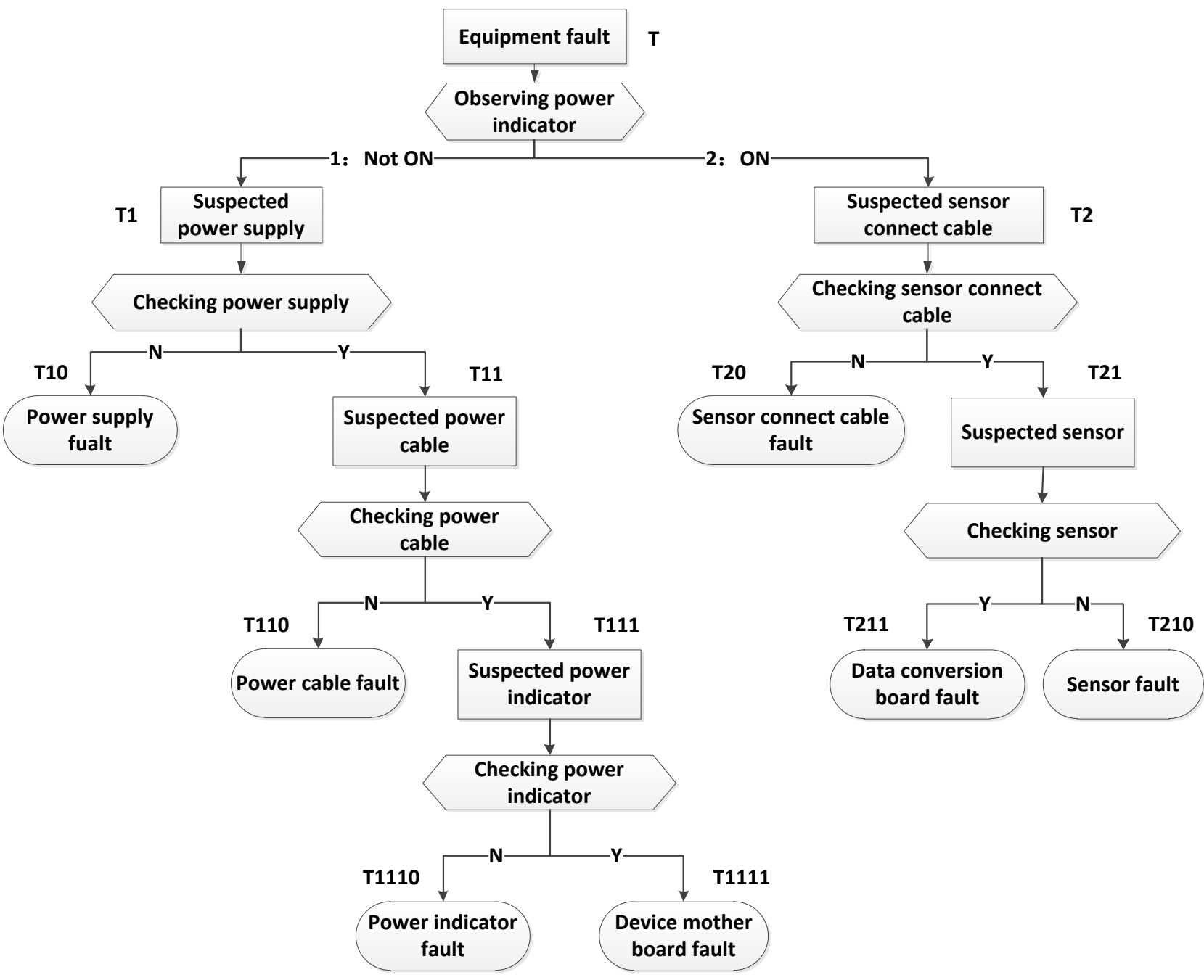

Figure 1. Equipment fault separation diagram

The device's internal card topology, devices, cable connection as shown in Fig. 2, if using fault tree in the process of fault isolation, every test requires manual inspection the card, device, cable inside the equipment, and efficiency is very low. While the equipment is working correctly, the 
working voltage of the board, device, cable inside of the equipment is within a certain range, so if the device is not working correctly, it will be reflected in the working voltage value. By embedding the data acquisition module in the equipment, we can real-time collect the voltage value and analyze the voltage value, then quickly to determine the source of failure. As shown in Fig. 3, acquisition voltage about the joints of the element of the equipment value of V1 V6, get the working voltage range of normal equipment from the equipment development unit, if it is within the normal range, expressed as 1 , if in the abnormal range, expressed as 0 , "*" expresses an arbitrary value. Such as: if output voltage V1 of power not in the normal range, then power failure, if $\mathrm{V} 1$ is in the normal range, then the power is normal; If the power supply is normal, if the output voltage V2 of power cable is abnormal, then power cable fault, if V2 is in the normal range, then the power cable is normal, and so on. This is shown in Table 1 equipment fault symptom list, according to the corresponding voltage value is normal or not, we can quickly to determine a component inside the equipment fault or not.

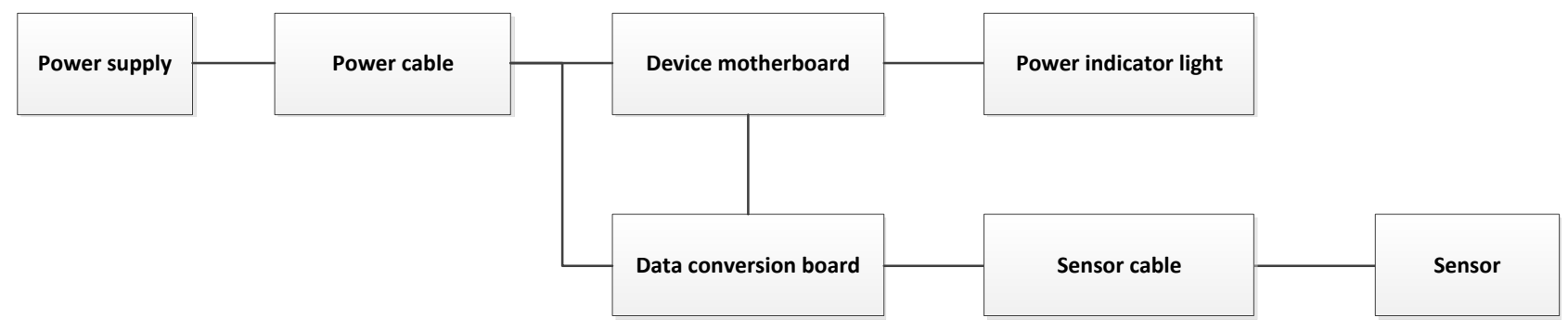

Figure 2. Equipment inner connection diagram

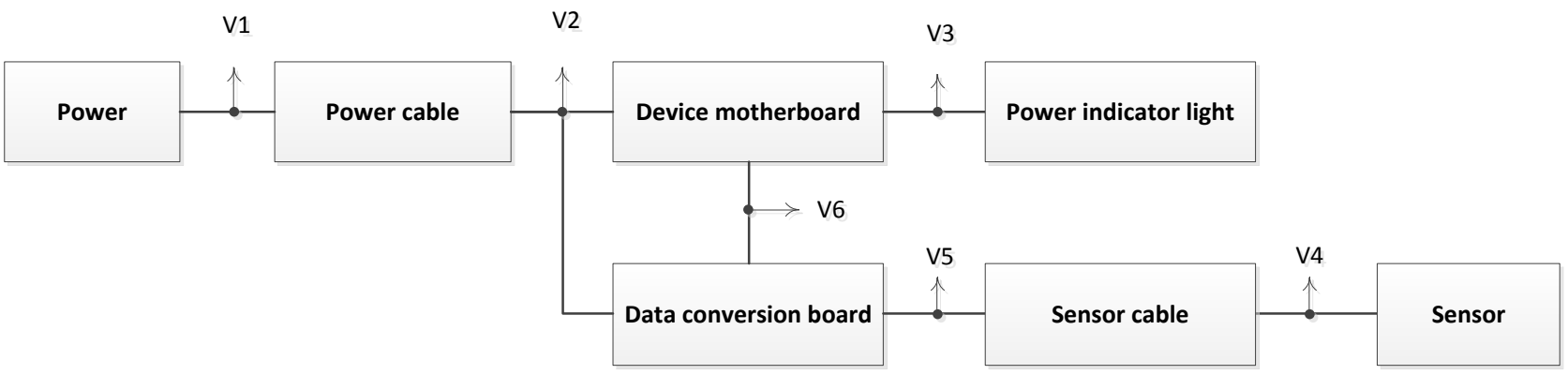

Figure 3. Voltage acquisition points of equipment

Table.1 Equipment fault symptoms list

\begin{tabular}{|c|l|l|l|l|l|l|l|}
\hline $\begin{array}{l}\text { Serial } \\
\text { number }\end{array}$ & V1 & V2 & V3 & V4 & V5 & V6 & The fault source \\
\hline 1 & 0 & $*$ & $*$ & $*$ & $*$ & $*$ & Power failure \\
\hline 2 & 1 & 0 & $*$ & $*$ & $*$ & $*$ & Power cable fault \\
\hline 3 & 1 & 1 & 0 & $*$ & $*$ & $*$ & Device motherboard failure \\
\hline 4 & 1 & 1 & 1 & 0 & $*$ & $*$ & Sensor fault \\
\hline 5 & 1 & 1 & 1 & 1 & 0 & $*$ & The sensor cable fault \\
\hline 6 & 1 & 1 & 1 & 1 & 1 & 0 & Data conversion board fault \\
\hline 7 & 1 & 1 & 1 & 1 & 1 & 1 & Normal \\
\hline
\end{tabular}

The equipment discussed in this section is used to analyze the diagnostic method of embedded fault diagnosis expert system, its internal structure is relatively simple, for complex equipment, we can also make fault diagnosis by a similar method, according to the development of equipment units to provide the connection diagram about internal elements of equipment, and the normal voltage 
range of the working point, then determine the group number of the voltage acquisition and the fault symptom list of corresponding equipment, in order to realize the real-time monitoring and diagnosis of equipment working state.

\section{System Design}

Embedded fault diagnosis expert system is divided into two parts the diagnosis Agent and client: diagnosis Agent directly connect with diagnostic equipment, real-time collect working state of equipment is diagnosed, and the data is sent to the client by the communication interface, then the client carries on the analysis to the data acquisition, using the reasoning machine of expert system to reasoning, diagnosis and quickly get diagnostic conclusion. The system architecture is shown in fig. 4.

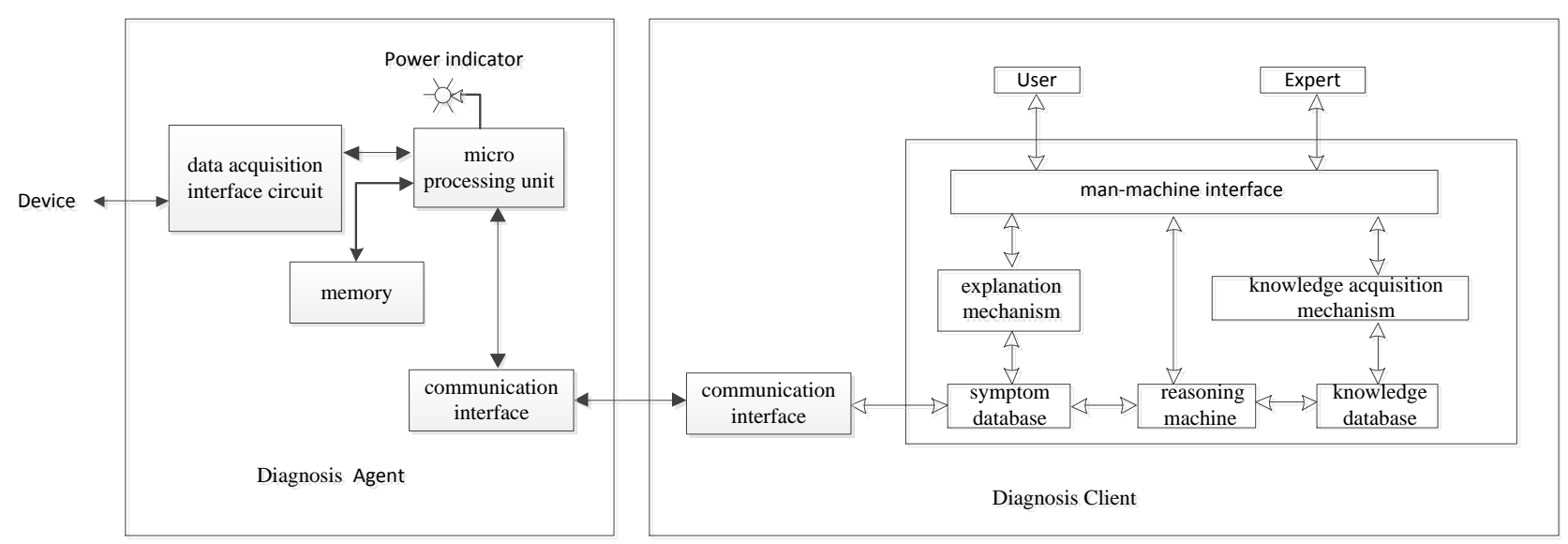

Figure 4. Embedded fault diagnosis expert system structure diagram

The diagnosis Agent: diagnosis Agent is composed of data acquisition interface circuit, the micro processing unit, a memory and a communication interface, and is embedded into equipment that is diagnosed to monitor the working state of the equipment. Diagnosis Agent real-time acquisition voltage of each monitoring point of equipment, its internal memory store the normal range of the voltage value, once collected value of voltage is transfinite that explain the equipment is not working properly. According to the fault symptom list as table 1 show to determining fault source of equipment. Diagnosis Agent is embedded into equipment is diagnosed, its volume must be very small, it is not has a huge knowledge base and display device, when the equipment is not working properly, diagnosis Agent through the alarm indicator (LED) send a fault message and will send the collected data to the communication interface.

Client: client consists of knowledge base, reasoning machine, knowledge acquisition mechanism, symptom database, man-machine interface, explanation mechanism, a communication interface ${ }^{[4]}$, for repair personnel repair equipment use, it can prompt the method of fault diagnosis, repair measures and others information and so on. Client is an embedded computer system with a relatively complete functions, its core is the sheer size of the knowledge base, the knowledge stored in the client in advance. In normal times client and diagnosis of Agent have not to connect, it is like a handheld PDA, the user can use the client to view or edit the knowledge. When an equipment failure, diagnosis Agent is embedded in the device can monitor the fault, and light alarm indicator lamp, at the time will need to client and diagnosis Agent connected through a communication interface, and receive the monitoring voltage value from the diagnosis Agent, repair personnel to carry out fault diagnosis based on monitoring the voltage and the diagnosis rules.

The knowledge in the knowledge base is divided into two categories: the first category is fault symptom list as shown in Table 1, following each group of signs (here refers to monitoring voltage value) can be accurately correspond to the fault source, according to the corresponding relationship can be quickly locate the fault source. But some equipment's internal structure is very complex, we may not be able to monitor working voltage of all working points, or only according to the working 
voltage could not be determine whether the equipment is working properly, at this time you need to use production rules to represent knowledge. The second kind of knowledge is the production rules, in the knowledge base preserved rules as the follows form: RULE (i) :IF Reality THEN Result ( $\mathrm{i}=$ $1,2, \cdots, N)$, RULE (i) called the i rule among the rule base ${ }^{[5]}$.

Such as: according to Fig. 3 to collect a group of voltage V1 V6, IF (V1 V6 voltage is normal, the indicator light does not shine) THEN (indicating lamp fault).

When reasoning machine to work, the first is use the first kind of knowledge to reasoning, by monitoring the voltage value from the diagnosis of Agent retrieval and matching items of the fault symptom list as shown in Table 1, if a match is found, namely find the fault source, then the diagnosis is complete; if you cannot find a match, then with the second kinds of knowledge, namely use production rules to reasoning: first take the initial fact data put into dynamic fact base, retrieving the fact premise of every rules among rule base, matching facts of dynamic fact base , then make the successful matching rules into constitute rule set for later use, and make the rules of rule set put into dynamic fact base as new facts, the diagnostic process according to the need for certain operations or test, and the test results also as new facts are added in the dynamic fact base, a reasoning process of production rules as shown in Fig. 5, By repeating this reasoning process, until find the fault source.

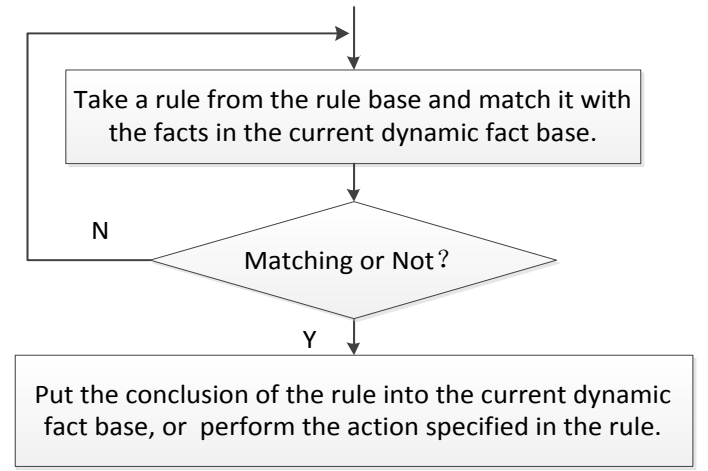

Figure 5. A reasoning process of production rules

\section{System Hardware Structure}

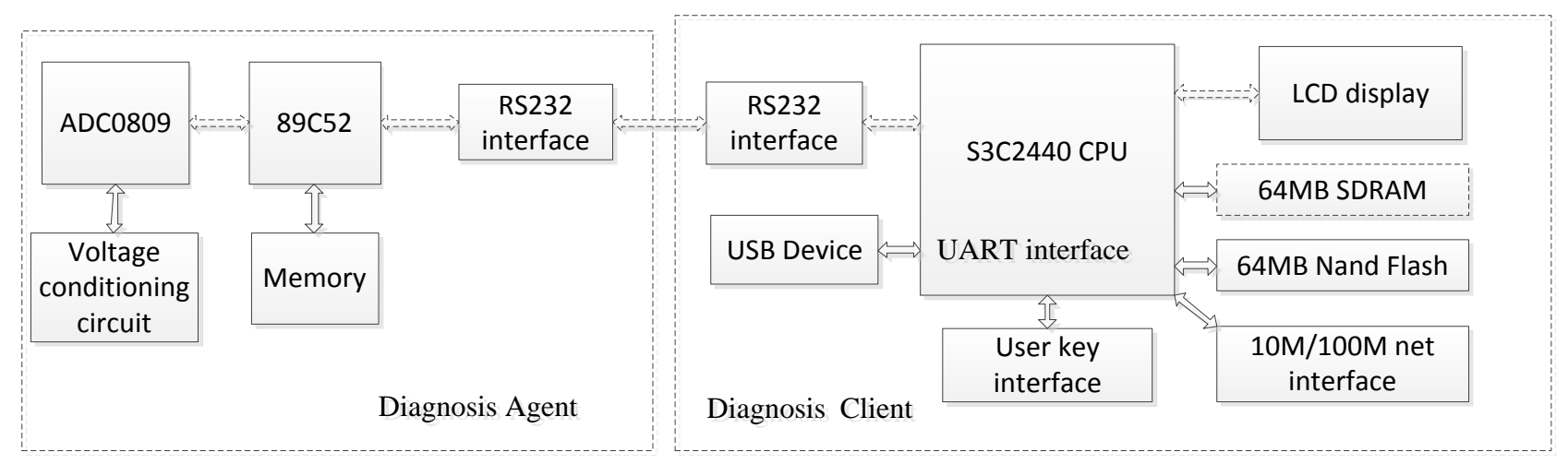

Figure 6. Schematic diagram of the system hardware

The hardware of the system consists of the diagnosis of Agent and client components, as shown in figure 6.

Diagnosis of Agent are composed of 89C52 MCU, memory, data acquisition module ADC0809, voltage conditioning circuit and RS232 interface, 89C52 through the ADC0809 collect the working voltage values of several working point of the equipment, those working voltage values are encoded, and send to the client by the RS232 interface. Most diagnosed working voltage values of the equipment are $+5 \mathrm{~V}$, for a voltage exceeding $+5 \mathrm{~V}$, need to be processed by voltage regulating circuit and then acquisition. 
Client consists of embedded computer system which is takes S3C2440 as the core, it includes the following parts:

1) Central Processing Unit: Use of core microprocessor of Samsung S3C2440, and its frequency of $200 \mathrm{MHz}$.

2) SDRAM; It is a 64M SDRAM, and clock frequencies up to $100 \mathrm{MHz}$, after system startup, program Operating into the memory.

3) NAND Flash: It is a 64M NAND Flash, when power down data is not easy to be lost, it is main storage the boot loader, and Linux kernel and root file system.

4) LCD display: Using 4.3 Inch high resolution Touch screen.

5) USB Device: When the system starts up use it downloaded program, there are main and Auxiliary two interfaces.

6) The user key interface: Received keystroke instructions from the user.

7) RS232 interface: For the data transmission between the client and the diagnosis of Agent.

8) $10 \mathrm{M} / 100 \mathrm{M}$ network interface: Data transmission when the system debugging, upgrade or knowledge base update.

Client receiving equipment condition data from the diagnosis of Agent through RS232 interface, after reasoning given detection method or repair steps, and presented to the user through the LCD display.

\section{System Software Design}

The system software is divided into two parts the diagnosis of Agent software and client software. Diagnosis of Agent software to realize data acquisition and communication, its function is relatively simple, this use of 51 series microcontroller through the $\mathrm{C}$ language completed. Diagnosis of client software to realize the expert knowledge base management, diagnostic reasoning, human-computer interaction, data communications and other functions, so its functional structure is very complex, the design is completed in the embedded Linux operating system, here mainly introduces the design of client software.

The Design of Knowledge Base. The knowledge base is the core of expert system, its main function is to make expert knowledge in a certain representation in the computer, and implement effective management on expert knowledge. The system uses the knowledge node structure as shown in Table 2 to preserve expert knowledge [6], and set up test knowledge items table and repair knowledge table in the SQLite database to store knowledge item.

Table.2 Frame structure of knowledge nodes

\begin{tabular}{ll}
\hline Content & instruction \\
The frame number & $\begin{array}{l}\text { Represent a note of signal fault network } \\
\text { Represent the fact or condition and node } \\
\text { attributes }\end{array}$ \\
Background knowledge item & $\begin{array}{l}\text { Represent fault symptoms are obtained } \\
\text { symptoms of knowledge item } \\
\text { Rules knowledge item }\end{array}$ \\
$\begin{array}{l}\text { Repair knowledge item } \\
\text { diagram knowledge item }\end{array}$ & $\begin{array}{l}\text { Represent of repair measures } \\
\text { Represent of diagram, schematic diagram etc. }\end{array}$ \\
\hline
\end{tabular}

The embedded Linux systems need to install and configure operation on the SQLite of the following: first download sqlite-3.6.16.tar.gz, and copy it to the under /tmp of the system directory, second, unzip the file bag, and enter the command tar zxvf sqlite-3.6.16.tar.gz, then switch to the /tmp/sqlite-3.6.16, and enter the ./configure, here can start the configuration, third, enter "make" began to compile, finally, enter "make install", "SQLite" is installed in the system[7]. We can Create SQLite database file, and create a data table according to the node structure, saving the knowledge to the corresponding data table, finally, complete the establishment of knowledge base. 
Communication Protocol. Diagnosis of Agent will transmit data to the client, the communication protocol between them as follows: "\$ID,1:xx.xx, 2:xx.xx, 3:xx.xx, 4:xx.xx, 5:xx.xx, 6:xx.xx, 7:xx.xx, 8:xx.xx,CHK:**\#",the "\$" as the start symbol, "ID" is the number of measured equipment, " 1:xx.xx" represent the first paths measured voltage values, by analogy, the total of the capable of measuring is 8 working value about the internal circuitry of the device;"CHK:**" for the frame data checksum, the sum of the frame data (not including the "CHK:**\#") ASCII value, "\#" as frame terminator. When the diagnosis of Agent in normal working, every second the voltage of the collected values are sent to the serial ports; after connecting the client and diagnosis of Agent, reading voltage value from the serial port as a basis for reasoning of fault diagnosis.

For different devices, also different for each voltage acquisition point, and the meaning of the parameters represent different, to the normal scope of each voltage value according to the specific equipment circuit structure, and corresponding to different voltage value system working state reflected, this information is stored in the knowledge base of expert system.

The Man-Machine Interface. Part of the man-machine interface is the main variety of interface design in expert system in the process of using, for example, the expert system operation interface based on the graphical user interface (GUI), knowledge base management interface.

In embedded Linux system, we can use Qt to develop efficient visualization program. Qt is a cross platform, object-oriented, C++ graphics application framework, Qt/Embedded is an embedded version of Qt, Qt/Embedded is based on the original Qt, and do a lot of good adjust to suitable for embedded environment. Qt Embedded by Qt API direct interaction with Linux I/O equipment, the interaction of Qt Embedded and Linux I/O by Qt API, and the object-oriented architecture makes the code structured, reusable and run fast ${ }^{[8]}$. The use of Qt to provide Designer tools, which is convenient for visual interface design, usability expert system. Qt provides specialized database access class, such as QSqlDatabase, QSqlQuery etc., the use of these functions can easily access the knowledge database.

After the program of expert system is debugged in the PC Linux environment, also to the program is transplanted to the embedded ARM-Linux platform work environment. In the cross compiler environment make the Qt project of the expert system to cross compile, and get the expert system program of the ARM-Linux version, then the expert system program of the ARM-Linux version is downloaded to the NAND Flash memory of client, and set the path of starting operation expert system application in the diagnosis of client system configuration file in /etc/init.d/rcS, restart the client again, the system will appear the client interface. When the equipment failure, the diagnosis of client and diagnosis of Agent connected with serial connection, Client will reasoning, diagnosis according to the equipment parameter diagnosis of Agent upload, combined with artificial analysis and judgments can quickly find the fault point equipment.

\section{Conclusions}

In this paper, the design of fault diagnosis expert system make the diagnosis of Agent and the client can be separated, diagnosis of Agent is embedded into weapons equipment to real-time acquisition working state, when the equipment failure, the diagnosis of Agent through the alarm indicator lamp gives the alarm signal, when repair personnel to repair, the diagnosis of client and diagnosis of Agent can be connected by communication cable, and repair personnel can real-time reading Fault information of the device, for online diagnosis of equipment, high efficiency, fast location of faults. This design enables the detection and repair equipment miniaturization, intelligent, easy to use repair personnel. Practice has proved, the use of embedded fault diagnosis expert system in weapon equipment repair and maintenance, playing a great role in promoting. The key technology of this system is that the data communication for diagnosis of Agent and diagnosis of client, if the communication mode change from the cable connection to the wireless network connection, it could not only bring convenience to repair personnel, also can realize distributed diagnosis, remote 
diagnosis and more others complex functions, to solve the problem of large complex equipment repair, and to provide a more reliable guarantee for the repair of weapon system.

\section{References}

[1] LI Xiang-yang, ZHANG Zhi-li, HUANG Xian-xiang, GAO Qin-he. Research on Simulation and Development of Fault Diagnosis Training Systemof Large-scale Armament Equipment [J]. Journal of System Simulation ,2009,21(21):6770

[2] Wang Feixuan,Wei Qingxin,Wang Kunming. Fault Diagnosis Development and Research on Weapon Equipment System [J]. Computer Measurement \& Control .2013,21 (4):977

[3] Li Jinghai. Research of Weapon Equipment Fault Diagnosis Technology [J]. Technology Foundation of National Defence,2008,3, (3):35

[4] Wang Xiao-yu , Peng Jiny-e, Wang Guo-qing. Analysis of Embedded On- Line Fault Diagnosis Expert System in Servo System [J]. Computer Measurement \& Control .2010,18 (3):498

[5] WANG Yong-qing. The principle and method of artificial intelligence [M]. Xi'an: Xi'an Jiaotong University Press, 2006.

[6] ZHAO Shi-feng, LIU Bai-lin. Knowledge Base Maintenance Method Based on Qt/Embedded and SQLite [J] Computer Knowledge and Technology, 2011, 7(35):9209.

[7] YU Hui. Embedded Linux System Design and Development Based on ARM [M]. Beijing: China Machine Press, 2010.

[8] SANG Jiang,CHEN Zhen. Application of embedded Linux system in PDA devices[J].Computer Engineering and Design,2005,26(4):1016 